\title{
Clinical Research: New Frontiers
}

Richard J. Barohn, MD - Chair, Department of Neurology; Gertrude and Dewey Ziegler Professor of Neurology; University Distinguished Professor; Director, Frontiers: The Heartland Institute for Clinical and Translational Research, Vice Chancellor for Research; President, Research Institute: University of Kansas Medical Center Laura Herbelin, BSc - Research Instructor, Department of Neurology, University of Kansas Medical Center Lauren S. Aaronson, PhD, RN, -Professor, School of Nursing and Dept. of Health Policy \& Management, School of Medicine; Deputy Director, Frontiers: The Heartland Institute for Clinical and Translational Research, University of Kansas Medical Center

linical research is undergoing significant changes due to both technological ad-
vances and new visions about clinical research by the NIH and PCORI, two
major funders of health related research. An often quoted statistic is that on
average new discoveries have taken 17 years to make their way into common practice.
All acknowledge this is far too long. Both NIH and PCORI recently have created and
funded large programs aimed at doing clinical research better and more efficiently so
that discoveries are brought to patient care and improve the health of the public more
rapidly. In 2006 and 2007 we provided initial information about the status of the long
standing General Clinical Research Center (GCRC) program supported by NIH for
over 40 years and the then newly launched Clinical and Translational Science Award
(CTSA) program designed to subsume and replace the GCRC program (Barohn, 2006;
Barohn and Aaronson, 2007). In this report we provide an update on these and other
programs that are aimed at improving clinical research as we know it.
First, we briefly discuss the CTSA and the PCORI Clinical Data Research
program funded by the National Center Network (CDRN) and identify several in-
for Advancing Translational Sciences dividual clinical research projects funded
(NCATS) at NIH and describe the current by PCORI. KUMC was awarded one of 11
status of Frontiers: The Heartland Institute CDRNs earlier this year, one of the au-
for Clinical and Translational Research, the thors of this paper (Barohn) is the princi-
CTSA program headquartered at KUMC. pal investigator of a specific comparative
Second, we discuss the NeuroNEXT pro- effectiveness clinical trial also funded by
gram, a consortium program funded by PCORI earlier this year, and KUMC in-
the National Institute of Neurological vestigators have been successful in ob-
Disorders and Stroke (NINDS), NIH, and taining additional clinical research grants
for which KUMC is one of 25 funded site from PCORI.
participants. Third, we discuss PCORI 
The Clinical and Translational Science Award (CTSA) Program

The CTSA programis a signature program of NIH (https://www.ctsacentral.org). With a budget of $\$ 475 \mathrm{M}$ annually it is the largest single program funded by NIH. Initially established under the former National Center for Research Resources, as identified above, it is now managed by the new National Center for Advancing Transla- (http://frontiersresearch.org). From its inception we were committed to advancing aspirations of making the Kansas City area a major hub for life sciences research. We reached out beyond KUMC and the University of Kansas Hospital to the other local academic health centers and their affiliated hospitals. Today, five academic institutions (KUMC, KU-Lawrence, KUMC-Wichita, UMKC, and

Table 1. The Frontiers network of academic and health-delivery institutions

\begin{tabular}{|l|l|}
\hline Academic partners & Health system partners \\
\hline University of Kansas Medical Center - Kansas & The University of Kansas Hospital, Kansas City, KS. \\
City & Wesley Medical Center, Wichita \\
University of Kansas Medical Center - Wichita & Via Christi Health, Wichita \\
University of Kansas - Lawrence & Veterans Administration Medical Center, Kansas City \\
University of Missouri-Kansas City & St. Luke's Health System, KS and MO \\
Kansas City University of Medicine & Children's Mercy Hospitals and Clinics KS and MO \\
and Biosciences & Truman Medical Center, Kansas City, MO. \\
& Swope Health Services, Kansas City, MO. \\
& Center for Behavioral Medicine, Kansas City, MO. \\
& Center for Practical Bioethics \\
\hline
\end{tabular}

tional Science (NCATS).

When first launched, the purpose of the CTSA program was to establish a transformative and integrated academic home for clinical and translational science. Each site was required to have a number of key function areas or cores and was allowed to propose unique novel methods programs drawing on strengths of the applicant organization. Funding formulas varied over the years since 2006 when the first CTSAs were funded. Frontiers: The Heartland Institute for Clinical and Translational Research was funded in June 2011 for $\$ 20 \mathrm{M}$ over five years and became part of what is now a 62 site national consortium.

Frontiers: The Heartland Institute for Clinical and Translational Research. The Frontiers program, headquartered at $\mathrm{KUMC}$, is a regional program
KCUMB) and 10 health care institutions (see Table 1) comprise the Frontiers program. Investigators from all of these sites are eligible to apply for and use Frontiers resources.

Among the successful programs under the Frontiers umbrella is a pilot study funding program. It is increasingly competitive to obtain extra-mural funding for research and having solid, supportive data from a pilot study has become essential for success with such funding. Since our initial funding in 2011, we have had four annual rounds of Requests for Proposals. Across these four years, we received 294 applications and funded 71 projects to investigators from Children's Mercy Hospital, KU-Lawrence, KUWichita, UMKC, and St. Luke's Health System, in addition to investigators from KUMC. Pilot study funding for a project has varied from $\$ 20-30 \mathrm{~K}$ for each project. 
For the Frontiers pilot study program we have used reviewers from across all Frontiers sites and have invited other units and programs that have pilot study funding to award to join our process for review and funding decisions. Each year we have modified our process in response to past years' experience. We now use an approach where the peer reviews of applications are taken to a funding council composed of representatives from the Frontiers sites and from other programs/units with pilot study funding to award. Combining the review and funding decisions of several programs has enhanced efficiency, reduced redundancy, and extended awareness and knowledge of the breadth of clinical and translational research across our institutions. The investment in these pilot studies has been substantial. For the 71 projects funded so far, $\$ 1.1 \mathrm{M}$ came from the CTSA grant and $\$ 685 \mathrm{~K}$ was contributed as 'cost-shared' funds from other programs (the KUMC Research Institute, the K-INBRE program, an American Cancer Society grant program at KUMC, the KUMC Diabetes Center and departments of internal medicine, neurology, and physical therapy \& rehabilitation sciences, KU-Wichita, UMKC, and Children's Mercy Hospital.)

While some of these pilot studies were recently funded and are currently ongoing, we already have seen substantial return on the investment of pilot study funding. Two R01s have been awarded by NIH to investigators for projects based on their pilot study work and one other pilot study recipient anticipates receiving funding for an R01 this fall. In addition, one NIH R03 has been awarded to an investigator who received Frontiers pilot study funding for preliminary work and one large PCORI grant has been awarded to another Frontiers pilot study recipient. These four funded projects amount to over $\$ 3.2 \mathrm{M}$ in extra-mural research funding.

In 2013 the Frontiers program launched another program to provide a smaller amount of funding on a more flexible basis than the annual call for the formal pilot study program. Dubbed the Trail Blazer program, this opportunity allows for obtaining that last bit of data needed for a competitive extra-mural research grant application or for an opportunity to capitalize on an existing funded project and extend its work toward an additional area of inquiry. These applications may be submitted at any time and undergo an administrative review. While applicants may ask for up to $\$ 5 \mathrm{~K}$, often less is requested or awarded. In its first 18 months, 45 applications were received for Trail Blazer awards and 33 were funded for a total investment of just over $\$ 88 \mathrm{~K}$. All Trail Blazer recipients commit to submitting an extra-mural grant application within 12 months. While it is still too early to see return on our investment in these Trail Blazer awards, two recipients used their Trail Blazer funding to extend work on their active NIH R01 grants.

Another major component of all CTSAs is education to train the next generation of the clinical and translational workforce. To this end, the Frontiers program established a Clinical and Translational Education Center (CTREC) to coordinate and manage several specific training programs. The CTREC currently of- 
fers a pre-doctoral clinical research training program (TL1), a post-doctoral mentored clinical research scholar program (KL2), and a post-doctoral fellows and junior faculty Clinical Research Curriculum Program. Trainees in each of these programs may complete a formal Masters in Clinical Research degree (MS-CR). The Clinical Research Curriculum Program primarily provides tuition assistance so that these post-doctoral fellows and junior faculty may take classes required for the MS-CR degree. Tuition costs also are covered for the TL1 trainees and the KL2 Scholars.

The TL1 program is a year-long program that provides institutional support to students in clinical doctoral programs who are seeking a practical introduction to clinical and translational research. Students selected for the TL1 program take this year out of their regular degree program, thus extending their training by one year. The Frontiers TL1 program has supported four students each year from the KU Schools of Medicine and Pharmacy, and from KCUMB.

The KL2 program offers institutional support for mentored career development of investigators who have recently completed professional training and are beginning a career in translational and/or clinical research. It is generally a twoyear program that involves a $75 \%$ time commitment with salary largely covered by the Frontiers CTSA grant. Commitments from the scholars' home departments were obtained to cover up to $25 \%$ salary so that each scholar would have the requisite $75 \%$ dedicated time for their research training and experience. This cost-sharing allowed us to support six
KL2 Scholars in each of the two cohorts of Scholars thus far. While the second cohort only recently started their training, two of the first six Scholars already have garnered independent funding for their research-one was awarded an R01from the National Cancer Institute at NIH and the other received a Blue KC Health Outcomes Grant.

The Frontiers program also provides other resources to support investigators. While we are not describing all of these in this paper, it is worth noting that Frontiers serves as a matchmaker for finding colleagues or mentors and provides a forum in which investigators may brainstorm their research ideas or get specific feedback on developing grant applications. This has shown to be a very valued resource. In the words of one investigator, recently funded by NIH for an R01, and who did not receive any specific funding assistance from Frontiers but did take advantage of our collegial assistance: "I can't sing the praises of the CTSA enough for the help it provided to us."

Network for Excellence in Neuroscience Clinical Trials (NeuroNEXT)

While the CTSA program is "disease agnostic", some of the categorical institutes at NIH have established national consortia specific to their institute's focus areas. NeuroNEXT is one such consortium or network supported by the National Institute of Neurological Disorders and Stroke (NINDS) (http://www.neuronext.org). It was created to expand the capability to conduct clinical studies in neuroscience. One of the authors of this paper (Barohn) is the principal investigator of the NeuroNEXT program at KUMC and chairs a 
formal CTSA/NeuroNEXT committee to promote synergy between these two programs.

The goals of NeuroNEXT are fourfold. The first goal is to test promising therapeutics in Phase II clinical trials, using biomarkers when available, and to generate results that may support moving forward with a larger Phase III trial using a Go/No-Go decision process. The second goal is to accelerate drug development through an established clinical trials infrastructure. In addition to funding the 25 sites that comprise the NeuroNEXT network, NINDS also funded a Clinical Coordinating Center at Mass General Hospital and a Data Coordinating Center at the University of Iowa to provide this clinical trial infrastructure. Through these entities, the NeuroNEXT network has the flexibility to take advantage of opportunities that emerge and to foster sharing of expertise in different diseases across the 25 network sites.

The third goal of the NeuroNEXT program is to decrease the cost of conducting trials and the time between trial design and trial completion. Two mechanisms that support this goal are the use of a central IRB and standard master trial agreements for all studies conducted through the NeuroNEXT program at the 25 sites in the network. The fourth goal is to coordinate efforts between the public and private sector and test the best therapeutics coming from both academic and industry investigators. To this end, NINDS leverages their existing relationships with academic investigators, industry investigators, and patient advocacy groups (Kearney, et al., 2014).
Through the NeuroNEXT program, KUMC is currently involved with four studies. These studies focus on four diseases. The first involves looking at biomarkers in spinal muscular atrophy. The other three are intervention trials involving multiple sclerosis, myasthenia gravis and stroke.

The PCORI Clinical Data Research Network (CDRN)

The Patient-Centered Outcomes Research Institute (PCORI) was established as part of the federal Affordable Care Act and authorized by Congress to fund and disseminate research on the best evidence available for patients and health care providers to make the best decisions about health care. It has a very substantial budget and has become a major player in the clinical research arena. One large program launched by PCORI is the PCORnet. The PCORnet is composed of both a clinical data research network (CDRN) and a patient powered research network. In this paper we address the CDRN program.

In January 2014, KUMC signed a \$7M contract with PCORI for the 18 month Phase 1 part of PCORnet as one of 11 funded CDRNs (R Waitman, KUMC, PI). Called The Greater Plains Collaborative (GPC), our CDRN involves seven states and 10 institutions (see Table 2) from Minnesota to Texas - all of which are home to the greater prairie chicken, our namesake and mascot. Nine of our 10 sites also are part of a local CTSA program. While some other CDRNs are at institutions with CTSAs, we believe the GPC has done the most to integrate and 
create synergy between these two large national programs.

The overall goal of PCORnet is "to improve the nation's capacity to conduct clinical research by creating a large, highly representative national patientcentered network that supports more efficient clinical trials and observational studies" (http://www.pcori.org). A key sent in hospitals and health care practices, there has been little quantitative evidence for determining the degree to which EHR data can be used to assess clinical effectiveness. Given the diversity of EHRs, there also are many challenges to establishing the interoperability and common language standards needed to conduct research across institutions us-

Table 2.The Greater Plains Collaborative (GPC) is a network of 10 leading medical centers in seven states.

\begin{tabular}{|l|l|}
\hline State & Medical Center \\
\hline Kansas & University of Kansas Medical Center \\
Missouri & Children's Mercy Hospital \\
lowa & University of lowa Healthcare \\
Wisconsin & $\begin{array}{l}\text { University of Wisconsin-Madison } \\
\text { Medical College of Wisconsin }\end{array}$ \\
& Marshfield Clinic \\
Minnesota & University of Minnesota Academic Health Center \\
Nebraska & University of Nebraska Medical Center \\
Texas & University of Texas Health Sciences Center at San Antonio \\
& University of Texas Southwestern Medical Center \\
\hline
\end{tabular}

concern for PCORI is involvement of patients and other stakeholders in all phases of clinical research involving peoplefrom idea conception to study design and implementation, data analyses and interpretation, and dissemination and implementation of treatments shown to be most efficacious. This is based in the PCORI commitment to supporting research "that will be useful to patients and other clinical decision makers by ensuring that their questions and concerns are the focus of our work." (Selby, et al, 2013).

The specific role of the CDRN program in PCORnet is to harness the rich data currently residing in electronic health records (EHRs). The GPC views the CDRN program as a test of the nation's multi-billion dollar investment in EHRs. While EHRs are increasingly pre- ing different EHRs. Technical issues involved in establishing the GPC and working with our 10 sites are discussed elsewhere (Waitman, et al., 2014).

While it is too soon to have any specific outcomes from the GPC to discuss here, we do want to identify that the GPC will be working within the PCORnet on three specific conditions. All applicants for a CDRN were required to select one common condition, one rare disease, and to agree to work on obesity as an allCDRN focus area. After consulting with our community stake-holders through our respective CTSA community engagement core function areas, the GPC selected breast cancer as our common condition. For our rare disease we selected amyotrophic lateral sclerosis (ALS), commonly known as Lou Gehrig's disease, 
because of the expertise we have in this specific rare disease.

Last, we introduce two independent projects recently funded by PCORI to illustrate the types of research that PCORI supports. Patient Assisted Intervention for Neuropathy: Comparison of Treatment in Real Life Situations (PAIN-CONTRoLS - R Barohn, PI) is comparing four different drugs used to treat neuropathic pain to see which is most effective. The other study, Smoking Cessation Versus Long-Term Nicotine Replacement among High-Risk Smokers (E Ellerbeck, PI) is comparing two different methods for smoking cessation in patients with chronic obstructive pulmonary disease (COPD.) Both studies are ongoing and both actively sought input and involvement of patients in designing the studies-resulting in changes and additions that made the studies more relevant to the patients affected by these conditions. These are just two examples of how this new funding resource is allowing our investigators to conduct practical comparative effectiveness studies in our region and to do so in partnership with the people whose health we hope to improve.

In sum, collectively, the CTSA program, the institute-specific research networks like the NeuroNEXT program, and PCORI for both its ambitious PCORNet program and its general portfolio of supported health outcomes research are driving a new frontier in clinical research. Central to this new frontier is collaboration across disciplines and institutions, within and outside academia, with industry and the public sector, including patients, advocates, and policy makers. The shared hope is that through such collaboration we can more rapidly and more rigorously find answers to the questions that matter most for achieving a healthier public.

\section{Acknowledgements:}

We would like to acknowledge funding by a Clinical and Translational Science Award from NCATS at NIH, UL1TR000001; a Clinical and Data Research Network award from PCORI as part of the PCORNet initiative, CDRN-130604631; a NeuroNEXT award from NINDS at NIH, U10NS077356; and a comparative effectiveness research project award from PCORI, CER-1306-02496.

\section{References}

Barohn RJ. Clinical Research Resources at the University of Kansas Medical Center: General Clinical Research Centers (GCRC) and Clinical Translation Science Awards (CTSA). The Merrill Advanced Studies Center, Report 2006; 110:51-60.

Barohn, RJ. and Aaronson, LS. The General Clinical Research Center (GCRC) and the Heartland Institute for Clinical and Translational Research (HICTR) at the University of Kansas Medical Center (KUMC), in Future Directions for Federal Research Funding, Merrill Series on the Research Mission of Public Universities, edited by Mabel Rice, University of Kansas, 2007; 111:32-43.

Kearney M, Ecklund D, Bosch M, Thornell B, McNeil D, Korshetz W, Kaufmann P, Coffey C, Cudkowicz M. Network for Excellence in Neuroscience Clinical Trials. NeuroNEXT: Increasing Efficiency in Clinical Trial Management. Neurology April 8, 2014 vol 82, no. 10 Supplement P1.334

Selby, J., Beal, A., Luce, B., and Yan, R.A strategic path to realizing our vision. PCORI 2013 Annual Report.

Waitman L.R., Aaronson L.S, Nadkarni P.M, Connolly D.W, and Campbell J.R. The Greater Plains Collaborative: APCORNet Clinical Research Data Network. J Am Med Inform Assoc. 2014; 21:637-641. PMID: 24778202 